\title{
On the angular momenta of galaxy structures
}

Piotr Flin ${ }^{1,2}$, W. Godłowski ${ }^{3}$ \& M. Szydłowski ${ }^{3}$

1. Pedagogical University, Institute of Physics, ul.Swietokrzyska 15, Kielce, 25-406, Poland

2. Bogoliubov Laboratory of Theoretical Physics, Joint Institute for Nuclear Research, Dubna, Moscow Region 141980, Russia

3. Astronomical Observatory of the Jagiellonian University, 30-244 Krakow, ul. Orla 171, Poland

Abstract. We discuss the possibility that angular momenta of galaxy structures are due to the global rotation of the Universe.

In homogeneous and isotropic models, the Universe may not only expand, but also rotate relative to the local gyroscope. The motion of the matter can be described by the Raychaudhuri equation relating the scalar equation $\Theta$, the rotation tensor $\omega_{a b}$ and the shear tensor $\sigma_{a b}$ (Li-Xin Li 1998):

$$
-\nabla_{a} A^{a}+\dot{\Theta}+\frac{1}{3} \Theta^{2}+2\left(\sigma^{2}-\omega^{2}\right)=-4 \pi G(\rho+3 p)
$$

where $\rho$ is the mass density, $p$ is the pressure, $A^{a}=u^{b} \nabla_{b} u^{a}$ is the acceleration vector, while $\omega^{2} \equiv \omega_{a b} \omega^{a b} / 2$ and $\sigma^{2} \equiv \sigma^{a b} \sigma_{a b} / 2$ are the scalar of rotation and sheer respectively. As the Universe expands $\sigma$ falls off more rapidly than the rotation $\omega$, so we consider the generalization of the Friedmann equation in which only the "centrifugal" term is present, i.e.

$$
\frac{\dot{a}^{2}}{2}+\frac{\omega^{2} a^{2}}{2}-\frac{4 \pi G a^{2}}{3 c^{2}} \epsilon=-\frac{k c^{2}}{2},
$$

where $\epsilon=\rho c^{2}, k$ is the curvature constant, $a$ a scalar factor and $\dot{a} \equiv \frac{d}{d t}$

Equation (2) should be completed with the principle of the conservation of energy-momentum tensor and angular momentum:

$$
\dot{\epsilon}=-(\epsilon+p) \Theta, \quad \Theta \equiv 3 \frac{\dot{a}}{a}, \quad \frac{p+\epsilon}{c^{2}} a^{5} \omega=J .
$$

The conservation of angular momentum of a structure relative to the gyroscopic frames in dust epochs gives:

$$
J=k M^{5 / 3}-l M
$$

where $k=\frac{2}{5}\left(\frac{3}{4 \pi \rho_{d 0}}\right)^{2 / 3} \omega_{0}, \rho_{d o}$ is the density of (dust) matter in the present epoch, and $l=\beta r_{f}^{2}\left(1+z_{f}\right)^{2} \omega_{0}, r_{f}$ is the radius of protostructure, $z_{f}$ redshift formation, and $\beta$ is a parameter determined by the distribution of the mass in 
it. We show (Godłowski et al. 2003) that relation (4) exhibits a clear minimum and the minimal mass of the structure

$$
M_{\min }=\left(\frac{3 l}{5 k}\right)^{3 / 2}=1.95 r_{f}^{3}\left(1+z_{f}\right)^{3} \rho_{d 0},
$$

does not depend on the present value of $\omega_{0}$ rotation of the Universe. For less and more massive structures it is predicted $|J(M)| \neq 0$. We assumed that galaxy clusters are formed through the collapse of the protostructures. For a protostructure with a diameter of $60 \mathrm{Mpc}$ and $z_{f}=6$, an estimated dust mass of a structure with vanishing angular momentum is $\approx 5 \times 10^{13} \mathcal{M}_{\odot}$. This gives the total mass of a structure of the order of $10^{14}-10^{15} \mathcal{M}_{\odot}$, which is the mass of a rich galaxy group.

We chose 18 galaxy groups in the LSC (Tully 1988), each containing more than 40 galaxies. The existence of alignment is interpreted as existence of non-vanishing angular momentum, so we checked the galaxy alignment in each group. The spatial orientation of a galaxy plane was determined considering both position and inclination angles of a galaxy (Flin \& Godlowski 1986). We determined the polar $(\delta)$ and azimuthal $(\eta)$ angles describing the location of the galaxy plane in supergalactic coordinates. The distributions of the supergalactic position angles $\mathrm{P}$ and the $\delta$ and $\eta$ angles were checked for isotropy using various statistical tests. In the case of Tully's groups we do not find any alignment.

The published results of galaxy alignment and spin investigations in pairs, yield the existence of non-vanishing angular momenta in these structures. There is evidence for alignment of galaxies within clusters and superclusters of galaxies. These findings taken together are in agreement with the considered models.

Our general conclusion is that the Li-Xin Li model, in which celestial bodies acquire angular momenta during their formation from the global rotation of the Universe, give us correct predictions, explaining why for galaxy groups contrary to the more massive structures no alignment is detected.

\section{References}

Flin, P., \& Godlowski, W. 1986, MNRAS, 222, 525

Godlowski, W., Szydlowski, M., Flin, P., \& Biernacka M. 2003, GRG, 35, 907

Li-Xin, L. 1998, GRG, 30, 497

Tully, R. B. 1988, Nearby Galaxies Catalog, Cambridge Univ. Press 